\title{
Genetic polymorphisms related to efficacy and overuse of triptans in chronic migraine
}

\author{
Giovanna Gentile $\cdot$ Marina Borro $\cdot$ Noemi Lala $\cdot$ \\ Serena Missori $\cdot$ Maurizio Simmaco • \\ Paolo Martelletti
}

Received: 23 April 2010/Accepted: 2 July 2010/Published online: 22 July 2010

(C) Springer-Verlag 2010

\begin{abstract}
Migraine is a common type of headache and its most severe attacks are usually treated with triptans, the efficacy of which is extremely variable. Several SNPs in genes involved in metabolism and target mechanisms of triptans have been described. To define an association between genetic profile and triptan response, we classified a migrainous population on the basis of triptan response and characterized it for polymorphisms in the genes coding for monoamine oxidase $\mathrm{A}, \mathrm{G}$ protein $\beta 3$ and the cytochrome CYP1A2. Analysis of the association between genotypic and allelic frequencies of the analyzed SNPs and the grade of response to triptan administration showed a significant correlation for MAOA uVNTR polymorphism. Further stratification of patients in abuser and non-abuser groups revealed a significant association with triptan
\end{abstract}

G. Gentile ( $\square)$

Department of Biochemical Sciences,

Advanced Molecular Diagnostic Unit,

2nd School of Medicine, Sant'Andrea Hospital,

Sapienza University of Rome,

Via di Grottarossa 1035, 00189 Rome, Italy

e-mail: giovanna.gentile@uniroma1.it

M. Borro - M. Simmaco

NESMOS Department,

Advanced Molecular Diagnostic Unit,

2nd School of Medicine, Sant'Andrea Hospital,

Sapienza University of Rome,

Via di Grottarossa 1035, 00189 Rome, Italy

N. Lala · S. Missori · P. Martelletti

Department of Medical and Molecular Sciences,

Regional Referral Headache Center,

2nd School of Medicine, Sant'Andrea Hospital,

Sapienza University of Rome,

Via di Grottarossa 1035, 00189 Rome, Italy overuse and, within the abusers, with drug response to the CYP1A2*1F variant.

Keywords Chronic migraine - SNPs - Triptan overuse · Triptan efficacy $\cdot$ Polymorphisms

\section{Introduction}

Migraine is a common type of headache and its most severe attacks are usually treated with triptans. The triptans are mainly used for the acute treatment of migraine attacks and their mechanism of action is based on the stimulation of serotonin receptors, yet their therapeutic benefits are largely variable among different subjects. Different metabolic rates may probably account for different levels of drug response.

Enzymes responsible for triptan degradation are the monoamine oxidase MAOA and the cytochrome P450: the former for sumatriptan and rizatriptan and the latter for eletriptan, frovatriptan and naratriptan. The two systems cooperate in the metabolism of almotriptan (MAO A and CYP3A4) and zolmitriptan (MAOA, CYP1A2 and CYP3A4) [1]. Several SNPs in these genes are related to alteration of enzymatic activity, and genotypization can thus contribute to define a specific metabolic background responsible for the drug's efficacy.

Polymorphisms in genes involved in transduction signal via the HT1B/1D receptor, as the C825T SNP in the gene coding the $G$ protein $\beta 3$ subunit (GNB3), can also be important determinants of triptan therapy outcome [2].

The aim of this work was to detect a correlation between triptan response and individual genetic profile, characterizing a panel of gene SNPs involved in the pharmacokinetics and pharmacodynamics of triptans in a chronic migrainous population. 


\section{Patients and methods}

Description of analyzed populations

The study received the approval of the University Ethic Committee. All patients signed a written consent before enrollment in the study.

A total of 150 patients (26 males and 124 females) affected with CM, aged 25-81 years, were enrolled in the study. All patients were referred to the Regional Referral Headache Centre of 2nd School of Medicine of Sapienza University at Sant'Andrea Hospital in Rome. The diagnosis of CM was performed according to the 2006 ICHD-II revised rules [3]. The majority of patients $(64.7 \%)$ were administered a single type of triptan $(12.7 \%$ sumatriptan, $19.4 \%$ eletriptan, $11.4 \%$ rizatriptan, $16.6 \%$ almotriptan, $4 \%$ frovatriptan, $0.6 \%$ zolmitriptan), while the remaining subjects $(35.3 \%)$ were administered different triptans.

Headache frequency and drug overuse were evaluated by means of headache diaries filled in for at least 3 months. The diagnosis of medication overuse headache (MOH) was performed according to the 2006 ICDH-II revised rules [4]. A total of 75 patients (16 males and 59 females) were triptan abusers, 51 were non-abusers and 24 patients were excluded because of the assignation to mixed abuser class (triptans plus other class of acute migraine drugs).

Acute therapy response was assessed by evaluating the following end points: 2 and $24 \mathrm{~h}$ pain free or of pain relief (relief $>50 \%$ of the VAS at baseline) plus the presence or not of recurrence. On this basis, the patients were categorized as follows: $0-25 \%$ response (class 1); 25-50\% response (class 2); 50-75\% response (class 3); 75-100\% response (class 4 ).

The distribution of response class and drug overuse in the studied population is reported in Table 1.

Table 1 Categorization of patients $(n=150)$ based on response grade and drug overuse of triptans

Patients no.

\begin{tabular}{ll}
\hline Therapy response grade & \\
$0-25 \%$ (class 1) & 38 \\
$25-50 \%$ (class 2) & 29 \\
$50-75 \%$ (class 3) & 35 \\
$75-100 \%$ (class 4) & 48 \\
Drug overuse & \\
Yes & 75 \\
No & 51 \\
Not assigned & 24 \\
\hline
\end{tabular}

Genetic analysis

Genomic DNA was isolated from peripheral blood using the X-tractor Gene system (Corbett Life Science, Australia). We studied the following DNA polymorphisms: an untranslated variable number of tandem repeats (uVNTR) of 30 base pairs located about $1.1 \mathrm{~kb}$ upstream of the ATG initiation codon of monoamine oxidase $\mathrm{A}$ (MAO A) gene; a $\mathrm{A}>\mathrm{C}$ substitution at position -163 and $\mathrm{a} \mathrm{G}>\mathrm{A}$ transition at position -3860 in the $5^{\prime}$ noncoding region of the CYP1A2 gene, indicated as $* 1 \mathrm{~F}$ allele (rs762551) and $* 1 \mathrm{C}$ allele (rs2069514), respectively, and a C $>\mathrm{T}$ transition at nucleotide 825 (rs5443) in the coding sequence of GNB3 gene, which produces a truncated form of the protein.

CYP1A2 and GNB3 polymorphisms were genotyped by pyrosequencing technology (Pyrosequencer PyroMark ID system-Biotage AB and Biosystems, Uppsala, Sweden), while MAOA uVNTR was evaluated by microchannel electrophoresis on chip (Bioanalyzer, Agilent technologies, Santa Clara, CA, USA), according to the protocols described in Gentile et al. [5].

Statistical analysis

Chi-square Hardy-Weinberg equilibrium (HWE) test calculator for biallelic markers available at http://www.oege. org/software/hwe-mr-calc.shtml [6] was used to test for deviations of genotype frequencies from HWE.

Chi-square analysis was applied to detect associations between the genotype/alleles and the therapy response class and to compare genotypic and allelic frequencies between abusers and non-abusers.

In the case of the X-linked MAOA uVNTR polymorphism, male patients (26 out of 150 total subjects) were excluded from statistical analysis. Values of $p<0.05$ were considered to be significant.

\section{Results}

All the polymorphisms analyzed in the total population were in HWE.

Genotype distributions were calculated for GNB3 and CYP1A2 in 150 migraineurs. In the case of the GNB3 C825T SNP, we found 68 (45.3\%) homozygous noncarriers of the mutated $825 \mathrm{~T}$ allele, $63(42 \%)$ heterozygous carriers and $19(12.7 \%)$ homozygous carriers of the mutated $825 \mathrm{~T}$ allele. The CYP1A $2 * 1 \mathrm{C}$ allele was found in $9(6 \%)$ heterozygous and $1(0.7 \%)$ homozygous carriers, the remaining $140(93.3 \%)$ subjects being homozygous noncarriers of the mutated $-3860 \mathrm{~A}$. The CYP1A2*1F allele was present in $61(40.7 \%)$ heterozygous carriers and 
$17(11.3 \%)$ homozygous carriers of the mutated $-163 \mathrm{C}$ allele, whereas $72(48 \%)$ subjects were homozygous noncarriers of the mutated $-163 \mathrm{C}$ allele. The MAOA uVNTR polymorphism was detected in the female population ( $n=124$ ) in the $3 \mathrm{R}, 4 \mathrm{R}$ and $5 \mathrm{R}$ variants, the $3 \mathrm{R}$ and $4 \mathrm{R}$ alleles accounting for $98.3 \%$ of the total. Since the $5 R$ variant was detected only in 4 patients out 150 , it was excluded from statistical analysis.

Association between genotype and allelic frequencies of the analyzed SNPs and the grade of response to triptan administration in the total population was assessed by Chi-square analysis.

A significant correlation was found only for MAOA uVNTR polymorphism, as shown in Table 2. In particular, compared to the expected values, we found an overrepresentation of $4 \mathrm{R} / 4 \mathrm{R}$ genotype and an underrepresentation of the $3 \mathrm{R} / 3 \mathrm{R}$ genotype in class $1(0-25 \%$ response) and 3 (50-75\%), whereas in class $2(25-50 \%)$ and 4 (75-100\%) we detected an increased number of $3 \mathrm{R} / 3 \mathrm{R}$ homozygotes and a decreased number of 4R/4R homozygotes $\left(\chi^{2}=18.4 ; d f=6 ; p=0.005\right)$. Similar results were obtained on analyzing allelic distribution: the number of $3 \mathrm{R}$ variant was decreased in class 1 and 3 and increased in class 2 and 4, while the 4R variant was overrepresented in class 1 and 3 and underrepresented in class 2 and 4 $\left(\chi^{2}=16.0 ; d f=3 ; p=0.001\right)$.

The migrainous population was then stratified according to drug overuse, and genotypic and allelic distributions were compared in abusers and non-abusers. All the polymorphisms analyzed in the stratified population were in HWE, except for CYP1A2*1C in the abuser group that gives a $p<0.05\left(\chi^{2}=7 ; d f=1\right)$ due to the presence of the rare AA homozygote.
The frequencies of MAOA, GNB3 and CYP1A2*1C variants were found not to be significantly different between abuser and non-abuser groups. In contrast, we found an increased number of $-163 \mathrm{C}$ allele of CYP1A 2 in the non-abuser class and an overrepresentation of $-163 \mathrm{~A}$ allele in the abuser group $\left(\chi^{2}=4.74 ; d f=1 ; p=0.029\right)$, as shown in Table 3. Hence, therapy response/genotype association was separately assessed for abusers and nonabusers. In the abuser group, a significant association with response grade was found for CYP1A2*1F allele (Table 4). In detail, A allele was overrepresented in the classes 1 and 2 and underrepresented in the classes 3 and $4\left(\chi^{2}=7.79\right.$; $d f=3 ; p=0.05)$. In the non-abuser group, there was no significant correlation of CYP1A2*1F allelic frequency and therapy response.

\section{Discussion}

We present the results of a pilot study aimed to explore the potentiality of pharmacogenomics to tailor CM therapy. Polymorphisms in genes involved in metabolism (CYP450 and MAOA) and in target mechanisms (GNB3) of triptans were genotyped in 150 chronic migraineurs to assess their relation with the efficacy of triptans.

Among the considered gene SNPs, no relation between genotype and/or allelic frequencies and response grade of triptans was found, except for MAOA uVNTR polymorphism.

Concerning the GNB3 gene, a previous study reported an association between the C825T SNP and the response to triptan in cluster headache $(\mathrm{CH})$ [2]. This polymorphism affects the signal transduction mechanism linked to

Table 2 Distribution of genotypic and allelic frequencies of MAOA uVNTR in the drug response classes assessed by Chi-test analysis

\begin{tabular}{|c|c|c|c|c|c|c|c|c|}
\hline \multirow[t]{3}{*}{ MAOA uVNTR genotype ${ }^{a}$} & \multicolumn{8}{|c|}{ Therapy response grade } \\
\hline & \multicolumn{2}{|c|}{$0-25 \%$ (class 1 ) } & \multicolumn{2}{|c|}{$25-50 \%$ (class 2) } & \multicolumn{2}{|c|}{$50-75 \%$ (class 3) } & \multicolumn{2}{|c|}{$75-100 \%$ (class 4$)$} \\
\hline & Obs. & Exp. & Obs. & Exp. & Obs. & Exp. & Obs. & Exp. \\
\hline 3R3R & 0 & 3.60 & 8 & 3.33 & 1 & 3.87 & 7 & 5.2 \\
\hline 3R4R & 12 & 11.2 & 10 & 10.4 & 10 & 12.1 & 18 & 16.2 \\
\hline $4 \mathrm{R} 4 \mathrm{R}$ & 15 & 12.2 & 7 & 11.2 & 18 & 13.1 & 14 & 17.6 \\
\hline \multirow[t]{2}{*}{ MAOA uVNTR alleles ${ }^{\text {b }}$} & \multicolumn{2}{|c|}{$0-25 \%$ (class 1 ) } & \multicolumn{2}{|c|}{$25-50 \%$ (class 2$)$} & \multicolumn{2}{|c|}{$50-75 \%$ (class 3) } & \multicolumn{2}{|c|}{$75-100 \%$ (class 4) } \\
\hline & Obs. & Exp. & Obs. & Exp. & Obs. & Exp. & Obs. & Exp. \\
\hline $3 R$ & 13 & 18.7 & 26 & 17.0 & 12 & 20.1 & 32 & 27.2 \\
\hline $4 \mathrm{R}$ & 42 & 36.3 & 24 & 33.0 & 47 & 38.9 & 48 & 52.8 \\
\hline
\end{tabular}

Statistical analysis was performed on 124 females. The 5R variant was excluded from statistical analysis

$d f$ degrees of freedom

a $\chi^{2}=18.4 ; d f=6 ; p=0.005$

b $\chi^{2}=16.0 ; d f=3 ; p=0.001$ 
Table 3 Distribution of allelic frequencies of CYP1A2 -163A $>$ C SNP in triptan abuser and non-abuser classes assessed by Chi-test analysis

\begin{tabular}{|c|c|c|c|c|}
\hline \multirow{2}{*}{$\begin{array}{l}-163 \mathrm{~A}>\mathrm{C} \\
(\mathrm{CYP} 1 \mathrm{~A} 2 * 1 \mathrm{~F}) \\
\text { alleles }\end{array}$} & \multicolumn{2}{|c|}{$\begin{array}{l}\text { Abusers }(n=75) \\
n(\%)\end{array}$} & \multicolumn{2}{|c|}{$\begin{array}{l}\text { Non-abusers }(n=51) \\
n(\%)\end{array}$} \\
\hline & Obs. & Exp. & Obs. & Exp. \\
\hline A & $108(72.0)$ & $100(66.6)$ & $60(58.9)$ & 68 (66.6) \\
\hline $\mathrm{C}$ & $42(28.0)$ & $50(33.4)$ & $42(41.2)$ & $34(33.4)$ \\
\hline
\end{tabular}

$\chi^{2}=4.74 ; d f=1 ; p=0.029$

$d f$ degrees of freedom

5-hydroxytryptamine (5-HT1B and 5-HT1D) receptors, the common targets of triptans [7]. In chronic migraine, we found no association of GNB3 C825T SNP with therapy response. However, $98.3 \%$ of the population studied by Schurks et al. was treated with sumatriptan, whereas our migraine population was heterogeneous with respect to the class of triptan medications. The discrepancy in the results probably reflects the distinct clinical presentation of different headaches as $\mathrm{CH}$ and $\mathrm{CM}$, as well as the multifactorial aspect of headache physiology and triptan pharmacokinetics. On the other hand, in CM we found some genotype-response correlation for MAOA uVNTR.

Monoamine oxidase $\mathrm{A}$ is the metabolic enzyme of neurotransmitters, and UVNTR polymorphism, located in the promoter region, affects its transcriptional activity modulating the levels of monoamines. In detail, enzyme expression is relatively high for carriers of 3.5 or 4 repeats (MAO A-high) and lower for carriers of 2, 3 or 5 repeats (MAO A-low) [8]. The 3R and 4R variants account for about $98 \%$ of the population we studied, and analysis of their frequencies in different response classes shows a peculiar, bimodal distribution where the long allele (higher enzymatic activity) is overrepresented in the classes 1-3 and underrepresented in classes $2-4$. The $4 \mathrm{R}$ homozygous genotype shows the same profile. Considering that high MAOA activity should faster metabolize the triptans, the increased number of $4 \mathrm{R}$ allele in class 1 may be expected, as well as its underrepresentation in class 4 . In contrast, the $4 \mathrm{R}$ frequency in classes 2 and 3 seems not to be consistent with the hypothesis that extensive metabolizers show poor therapy response, giving rise to the possibility of additional interacting factors affecting the phenotype.

A more clear and interesting link between drug response and genetic profile emerged stratifying patients in abuser and non-abuser groups, in the case of the CYP1A2*1F. The cytochrome $\mathrm{P} 450$ enzyme CYP1A2 plays a main role in the metabolism of a variety of structurally unrelated compounds, including a broad range of different drugs, and its activity is widely modulated by a plethora of inducer/ inhibitor molecules. As the other members of the cytochrome P450 superfamily, CYP1A2 gene is highly polymorphic and the $* 1 \mathrm{~F}$ allele is associated with reduced metabolic activity. In particular, $-163 \mathrm{C}$ allele presents reduced enzymatic activity compared to the $-163 \mathrm{~A}$ one, which also shows enhanced inducibility by environmental and chemical factors [9]. In relation to CYP1A2 pharmacokinetics, a higher enzymatic activity should determine faster rate of drug degradation, thus lowering drug concentration below the therapeutic threshold. The higher frequency of $-163 \mathrm{~A}$ allele in the abuser compared to nonabuser group may be explained by a reduced availability of the assumed triptan, leading to drug overuse. This assumption is supported by the finding that inside the abuser group, the higher activity allele $-163 \mathrm{~A}$ is also associated with low grade of therapy response (class 1 and 2). Hence, the $-163 \mathrm{~A}$ carriers, having a lower drug availability, attain limited therapeutic effect and overuse of drug.

In conclusion, our data suggest that individual genetic traits could in effect contribute to determine the treatment outcome of triptans, which frequently prompts drug misuse and overuse [10, 11]. However, the complexity of CM pathophysiology, still poorly characterized, and the incomplete definition of molecular pathways involved in the action and kinetics of triptans make it difficult to recognize genetic determinants strong enough to achieve reliable prevision of the efficacy of therapy $[12,13]$. Further, reproducibility and validation of genetic association studies in the research area of CM management strongly need standardized and more accurate methods for unambiguous

Table 4 Distribution of allelic frequencies of CYP1A2 -163A > C SNP in the drug response classes assessed by Chi-test analysis

\begin{tabular}{|c|c|c|c|c|c|c|c|c|}
\hline \multirow{3}{*}{$\begin{array}{l}-163 \mathrm{~A}>\mathrm{C} \\
(\mathrm{CYP} 1 \mathrm{~A} 2 * 1 \mathrm{~F}) \text { alleles }\end{array}$} & \multicolumn{8}{|c|}{ Therapy response grade } \\
\hline & \multicolumn{2}{|c|}{$0-25 \%$ (class 1) } & \multicolumn{2}{|c|}{$25-50 \%$ (class 2$)$} & \multicolumn{2}{|c|}{$50-75 \%$ (class 3) } & \multicolumn{2}{|c|}{$75-100 \%$ (class 4) } \\
\hline & Obs. & Exp. & Obs. & Exp. & Obs. & Exp. & Obs. & Exp. \\
\hline $\mathrm{A}$ & 29 & 27.4 & 25 & 20.2 & 15 & 18.7 & 39 & 41.8 \\
\hline $\mathrm{C}$ & 9 & 10.6 & 3 & 7.84 & 11 & 7.28 & 19 & 16.2 \\
\hline
\end{tabular}

$\chi^{2}=7.79 ; d f=1 ; p=0.050$

$d f$ degrees of freedom 
classification of therapy efficacy, till now based on patient self-reports [14, 15].

Conflict of interest GG, MB, NL and MS have declared no conflict of interest. SM won an unconditioned 2 years university fellowship funded by Merck Sharp \& Dohme. PM has received honoraria for contribution to advisory boards and research projects, and participation in clinical trials from Abbott, Allergan, ACRAF, Guidotti, Lusofarmaco and Merck Sharp \& Dohme. PM has also received unconditioned educational funds for young researcher fellowships from the University Department of ACRAF, Allergan, Bayer, Lusofarmaco and Merck Sharp \& Dohme.

\section{References}

1. Ferrari A, Sternieri E, Ferraris E, Bertolini A (2003) Emerging problems in the pharmacology of migraine: interactions between triptans and drugs for prophylaxis. Pharmacol Res 48:1-9

2. Schürks M, Kurth T, Stude P, Rimmbach C, de Jesus J, Jonjic M, Diener HC, Rosskopf D (2007) G protein beta3 polymorphism and triptan response in cluster headache. Clin Pharmacol Ther 82:396-401

3. Headache Classification Committee, Olesen J, Bousser MG, Diener HC, Dodick D, First M, Goadsby PJ, Göbel H, Lainez MJA, Lance JW, Lipton RB, Nappi G, Sakai F, Schoenen J, Silberstein SD, Steiner TJ (2006) New appendix criteria open for a broader concept of chronic migraine. Cephalalgia 26:742-746

4. Silberstein SD, Olesen J, Bousser MG, Diener HC, Dodick D, First M, Goadsby PJ, Göbel H, Lainez MJ, Lance JW, Lipton RB, Nappi G, Sakai F, Schoenen J, Steiner TJ, International Headache Society (2005) The International Classification of Headache Disorders, 2nd edn (ICHD-II)—revision of criteria 8.2 Medication overuse headache. Cephalalgia 25:460-465
5. Gentile G, Missori S, Borro M, Sebastianelli A, Simmaco M, Martelletti P (2010) Frequencies of genetic polymorphisms related to triptans metabolism in chronic migraine. $\mathrm{J}$ Headache Pain 11:151-156

6. Rodriguez S, Gaunt TR, Day INM (2009) Hardy-Weinberg equilibrium testing of biological ascertainment for Mendelian randomization studies. Am J Epidemiol 169:505-514

7. Siffert W, Rosskopf D, Siffert G, Busch S, Moritz A, Erbel R, Sharma AM, Ritz E, Wichmann HE, Jakobs KH, Horsthemke B (1998) Association of a human G-protein beta3 subunit variant with hypertension. Nat Genet 18:45-48

8. Sabol SZ, Hu S, Hamer D (1998) A functional polymorphism in the monoamine oxidase A gene promoter. Hum Genet 103:273-279

9. Sachse C, Brockmoller J, Bauer S, Roots I (1999) Functional significance of a $\mathrm{C} \rightarrow$ A polymorphism in intron 1 of the cytochrome P450 CYP1A2 gene tested with caffeine. Br J Clin Pharmacol 47:445-449

10. Di Lorenzo C, Di Lorenzo G, Sances G, Ghiotto N, Guaschino E, Grieco GS, Santorelli FM, Casali C, Troisi A, Siracusano A, Pierelli F (2009) Drug consumption in medication overuse headache is influenced by brain-derived neurotrophic factor Val66Met polymorphism. J Headache Pain 10:349-355

11. Hagen K, Jensen R, Bøe MG, Stovner LJ (2010) Medication overuse headache: a critical review of end points in recent followup studies. J Headache Pain. doi:10.1007/s10194-010-0221-4

12. Antonaci F, Dumitrache C, De Cillis I, Allena M (2010) Review of current European treatment guidelines for migraine. J Headache Pain 11:13-19

13. Bigal ME, Ho TW (2009) Is there an inherent limit in the migraine acute treatment efficacy? J Headache Pain 10:393-394

14. Stovner LJ, Tronvik E, Hagen H (2009) New drugs for migraine. J Headache Pain 10:395-406

15. Tfelt-Hansen P (2009) What can be learned from the history of recurrence of migraine? A comment. J Headache Pain $10: 311-315$ 